\title{
Locus-specific DNA methylation of Mecp2 promoter leads to autism-like phenotypes in mice
}

\author{
Zongyang Lu ${ }^{1,2,3}$, Zhen Liư ${ }^{4}$ Wei Mao ${ }^{5}$, Xinying Wang ${ }^{5}$, Xiaoguo Zheng ${ }^{1}$, Shanshan Chen ${ }^{4}$, Beibei Cao ${ }^{5}$, \\ Shisheng Huang ${ }^{1}$, Xuliang Zhang ${ }^{1}$, Tao Zhou ${ }^{1}$, Yu Zhang $\mathbb{1}^{1}$, Xingxu Huang ${ }^{1,2}$, Qiang Sun ${ }^{4}$ and Jia-Da Li ${ }^{5}$
}

\begin{abstract}
Autism spectrum disorder (ASD) is a neurodevelopmental disease with a strong heritability, but recent evidence suggests that epigenetic dysregulation may also contribute to the pathogenesis of ASD. Especially, increased methylation at the MECP2 promoter and decreased MECP2 expression were observed in the brains of ASD patients. However, the causative relationship of MECP2 promoter methylation and ASD has not been established. In this study, we achieved locus-specific methylation at the transcription start site (TSS) of Mecp2 in Neuro-2a cells and in mice, using nuclease-deactivated Cas9 (dCas9) fused with DNA methyltransferase catalytic domains, together with five locus-targeting sgRNAs. This locus-specific epigenetic modification led to a reduced Mecp2 expression and a series of behavioral alterations in mice, including reduced social interaction, increased grooming, enhanced anxiety/depression, and poor performance in memory tasks. We further found that specifically increasing the Mecp2 promoter methylation in the hippocampus was sufficient to induce most of the behavioral changes. Our finding therefore demonstrated for the first time the casual relationship between locus-specific DNA methylation and diseases symptoms in vivo, warranting potential therapeutic application of epigenetic editing.
\end{abstract}

\section{Introduction}

Autism spectrum disorder (ASD) represents a group of neurodevelopmental diseases characterized by defective social interaction and stereotypic behaviors. It is highly heritable, but has great genetic and clinical heterogeneity. More than 700 risk genes have been identified for ASD, but the effect of each gene is quite small for the great majority of them ${ }^{1,2}$. Accumulating evidence suggests that epigenetic factors may also play an important role in ASD pathogenesis ${ }^{3}$. For instance, there is considerable discordance in ASD-related behaviors between the

\footnotetext{
Correspondence: Xingxu Huang (huangxx@shanghaitech.edu.cn) or Qiang Sun (qsun@ion.ac.cn) or Jia-Da Li (lijiada@sklmg.edu.cn)

${ }^{1}$ School of Life Science and Technology, ShanghaiTech University, 100 Haike Rd., Pudong New Area, Shanghai 201210, China

${ }^{2}$ CAS Center for Excellence in Molecular Cell Science, Shanghai Institute of Biochemistry and Cell Biology, Chinese Academy of Sciences, University of Chinese Academy of Sciences, 320 Yueyang Road, Shanghai 200031, China Full list of author information is available at the end of the article. These authors contributed equally: Zongyang Lu, Zhen Liu, Wei Mao Edited by D. Heery
}

monozygotic twins ${ }^{4}$. Furthermore, epigenetic modification involving DNA methylation has been identified in ASD patients for several ASD-associated genes, such as $M E C P 2, F M R 1$, and $S H A N K 3^{5-10}$.

The gene MECP2 encodes methylated CpG-binding protein 2 (MeCP2), a putative transcriptional repressor that binds to methylated CpGs. Loss of MECP2 results in Rett syndrome (RTT) ${ }^{11}$, and patients with RTT exhibit a broad range of impairment in social behaviors, cognition, and coordination. Recently, mutations in MECP2 have also been identified in sporadic ASD patients ${ }^{12}$. Furthermore, increased $M E C P 2$ promotor methylation and decreased $M E C P 2$ expression were observed in the brain of ASD patients ${ }^{7,8}$, but their causative role in the ASD pathogenesis remains to be clarified.

In this study, we examined the role of Mecp 2 promoter methylation in ASD pathogenesis by using an epigenetic editing strategy. First, we developed an epigenome-editing approach using nuclease-deactivated Cas9 (dCas9) fused with DNA methyltransferase catalytic domains, together

\section{(c) The Author(s) 2020}

(c) (i) Open Access This article is licensed under a Creative Commons Attribution 4.0 International License, which permits use, sharing, adaptation, distribution and reproduction cc) in any medium or format, as long as you give appropriate credit to the original author(s) and the source, provide a link to the Creative Commons license, and indicate if changes were made. The images or other third party material in this article are included in the article's Creative Commons license, unless indicated otherwise in a credit line to the material. If material is not included in the article's Creative Commons license and your intended use is not permitted by statutory regulation or exceeds the permitted use, you will need to obtain permission directly from the copyright holder. To view a copy of this license, visit http://creativecommons.org/licenses/by/4.0/. 
with five locus-targeting sgRNAs. We then demonstrated its reliability in specifically increasing the methylation at Mecp2 promoter using Neuro-2a cell cultures. We further generated mice with specific methylation at this TSS and demonstrated that its methylation down-regulated Mecp2 expression and induced autism-like behaviors. Finally, we found that specifically increasing the Mecp 2 methylation in the hippocampus is able to induce most of the behavioral alteration.

\section{Materials and methods Animals}

All the experimental procedures were approved by the Institutional Animal Care and Use Committee (IACUC) of School of Life Sciences of Central South University, Changsha, China.

\section{Plasmid design and construction}

The pST1374-CMV-Cas9-NLS plasmid (Addgene, 44758) was used as the backbone for construction. Briefly, the CMV promotor was replaced by an EF1 $\alpha$ promotor at first. In order to generate DNMT3L-DNMT3A single-chain fusion protein, we have introduced the C-terminal domain of human DNMT3L (amino acids M178-S380) to the Nterminus of the catalytic domain of human DNMT3A (amino acids P627-V912), with a 16-amino acid linker (SSGRSFSSGLVPRGSH). The DNMT3L-DNMT3A was then fused to the N-terminus of dead Cas9 (dCas9). Dead DNMT3A mutation (C710S in human DNMT3A) and dead Cas9 mutation (D10A, H840A, and N863A in Cas9) were introduced using Mut-Express II Fast Mutagenesis Kit V2 (Vazyme) following manufacturer's instructions. The oligonucleotides for sgRNAs were synthesized, annealed, and cloned into the Bsa I site in the pGL3-sgRNA vector as described previously ${ }^{13,14}$. For AAV-based methylation vectors, the same sequence of DNMT3A was fused to the Nterminus of dCas9, and dCas9 was split into two parts between amino acids E573 and C574. The sequences for primers were listed in Table S3.

\section{Cell culture and transfection}

Neuro-2a (N2a) cells (from male mice) were purchased from ATCC (ATCC, CCL-131) without mycoplasma contamination and cultured in DMEM containing 10\% FBS in $37^{\circ} \mathrm{C}$ under $5 \% \mathrm{CO}_{2}$ atmosphere. Twenty six cells were transfected using Lipofectamine 2000 reagent (Life Technologies) according to manufacturer's protocols.

\section{T7ENI cleavage assay and sequencing}

Cells were harvested with lysis buffer $(10 \mu \mathrm{M}$ Tris-HCl, $0.4 \mathrm{M} \mathrm{NaCl}, 2 \mu \mathrm{M}$ EDTA, $1 \%$ SDS and $100 \mu \mathrm{g} / \mathrm{ml}$ Proteinase $\mathrm{K}$ ) at $55^{\circ} \mathrm{C}$ for $6 \mathrm{~h}$. Genomic DNA was extracted by phenol-chloroform and isopropyl alcohol precipitation according to standard procedures.
The editing efficiency was estimated by T7 endonuclease I assay. Briefly, the TSS region of Mecp 2 were PCRamplified from genomic DNA and the products were purified by cleanup kit (Axygen) according to the manufacturer's protocol. The sequences for primers were listed in Table S3. Purified PCR products were denatured in NEB buffer2 (NEB), and then digested with T7ENI (Vazyme) for $30 \mathrm{~min}$. Digested PCR products were resolved on a $2 \%$ agarose gel. PCR-amplified fragments were also ligated into pMD19T vector (Takara). After transformation, colonies with ligation products were sequenced for analyzing the editing efficiency.

\section{Immunofluorescent staining}

Mice were trans-cardially perfused with $4 \%$ paraformaldehyde (PFA), and brains were dissected into $4 \%$ PFA for post-fixation overnight. Following dehydration in $30 \%$ sucrose, brains were sectioned at $30 \mu \mathrm{m}$ and subjected to immunofluorescence staining. Briefly, floating cryosections were washed in PBS, and then incubated overnight with primary antibody (rabbit anti-MeCP2, 1:1000; cell signaling, $3456 \mathrm{~S}$ ) diluted in blocking buffer (1.5\% BSA, $0.1 \%$ Triton-X, 5\% NGS, pH7.4, $1 \times \mathrm{PBS}$ ). After three washes in PBST (0.1\% Triton-X, pH7.4, $1 \times$ $\mathrm{PBS}$ ), sections were incubated for $2 \mathrm{~h}$ with the secondary antibody (594-AffiniPure Donkey Anti-Rabbit IgG $(\mathrm{H}+\mathrm{L})$, 1:1000; Jackson, 711-585-152). Hoechst 33258 (1:5000; Yeasen) was used for nuclei staining. After three washes in PBST, samples were mounted on the glass slices. Images were captured using a Zeiss Axioimager Z2 microscope and processed using the Image J Fiji software.

\section{Western blot}

Brains were dissected into ice-cold PBS, the hippocampus and parietal cortex were rapidly dissected out. Tissue samples were lysed with $500 \mu \mathrm{l}$ ice-cold RIPA buffer (Beyotime Biotechnology) containing $1 \mathrm{mM}$ protease inhibitor PMSF (Beyotime Biotechnology) and PIC (Sigma). Cell lysates were homogenized by a homogenizer (PRO Scientific, Inc.) and the protein concentration was determined using a BCA Protein Assay Kit (Thermofisher, 23227). Protein lysates were dissolved in SDS-PAGE protein loading buffer and heated at $95^{\circ} \mathrm{C}$ for $10 \mathrm{~min}$. Samples were separated on 10\% SDS-PAGE gels (Epizyme, Lnc) and transferred onto PVDF membranes (Immobillon). After blocking with TBST solution containing 5\% skimmed milk for $1 \mathrm{~h}$ at room temperature, membranes were incubated with primary antibodies (rabbit anti-MeCP2, 1:2000; cell signaling, 3456S; mouse anti-Actin, 1:5000; Sigma, A4700-2ML) followed by HRP-conjugated secondary anti-rabbit and anti-mouse antibodies (1:5000; Vector lab). Blots were imaged with Amersham Imager 600 and quantified using the Image J Fiji software. 


\section{RT-qPCR}

Total RNA was prepared from cultured cells or tissues using TransZol Up Plus RNA Kit (TransGen). RNA was converted to cDNA using RT Master Mix (Takara, RR036A). Gene expression levels were measured with ViiA7 Real-Time PCR System (Applied Biosystems) using SYBR qPCR Master Mix (Vazyme) according to the manufacturer's instructions. Primer information was described in Table S3.

\section{Mouse zygote injection and embryo transfer}

Female C57BL/6 mice (4-week-old) were super-ovulated and mated to C57BL/6 male mice. Zygotes were collected from oviducts of the female mice. For zygotes injection, well mixed plasmids $(25 \mathrm{ng} / \mu \mathrm{l} \quad 5 \mathrm{U} 6 \quad$ sgRNAs $+50 \mathrm{ng} / \mu \mathrm{l}$ DNMT3L-DNMT3A-dCas9 or $50 \mathrm{ng} / \mu \mathrm{l}$ DNMT3LDNMT3A-mut- dCas9) were injected into the cytoplasm of zygotes in a droplet of M2 medium containing $5 \mu \mathrm{g} / \mathrm{ml}$ cytochalasin B (CB) using a piezo (Primetech) microinjector. The injected zygotes were cultured in KSOM medium at $37{ }^{\circ} \mathrm{C}$ under $5 \% \mathrm{CO}_{2}$ and transferred to oviducts of pseudopregnant ICR females at $0.5 \mathrm{dpc}$.

\section{Behavior test overview}

Male mice at the age of 3-18 weeks were used for all tests. The mice were transferred to the animal facility 2 weeks before the behavioral tests. Mice was allowed to habituate to the testing room for at least $30 \mathrm{~min}$. Three chamber, EPM, open field tests were measured by automated software. STFP, novel objective recognition, tail suspension, and self-grooming tests were scored manually.

\section{Social interaction tests}

Three-chamber test: The three-chamber test was performed as described previously ${ }^{15}$. The apparatus was comprised of a rectangular box $(70 \mathrm{~cm} \times 36 \mathrm{~cm} \times 30 \mathrm{~cm})$ divided into three chambers with partitions. The test mouse was placed in the closed middle chamber to habituate for $5 \mathrm{~min}$. Each side chamber contained an empty wire cage. After habituation, one stranger mouse (stranger 1, 10-12-weekold male) was introduced to the wire cage on one side. Another empty cage contained an inanimate object. The test mouse was allowed to explore the entire apparatus for $10 \mathrm{~min}$, and time spent in each chamber was recorded. A new stranger 2 mouse (10-12-week-old male) was introduced to the cage containing an inanimate object before. The test mouse spent another $10 \mathrm{~min}$ in exploring the entire apparatus and time spent in each chamber was recorded.

\section{Motor, anxiety-, and depression-related behavioral tests}

Open field: Locomotor activity was assessed over $10 \mathrm{~min}$ in an open field apparatus $(38 \mathrm{~cm} \times 38 \mathrm{~cm} \times 38 \mathrm{~cm})$. Locomotor activity was evaluated through total distance traveled by each mouse.
Elevated plus maze: The elevated plus maze (EPM) was comprised of two close arms $(26 \mathrm{~cm} \times 7 \mathrm{~cm})$ and two open arms $(26 \mathrm{~cm} \times 7 \mathrm{~cm})$ elevated $50 \mathrm{~cm}$ above the floor. Mice were placed in the center facing close arms and allowed to explore for $10 \mathrm{~min}$. Anxiety-like behavior was assessed by time traveled in the open arms.

Tail suspension: Mice were gently suspended by the tail. Depression behavior was assessed over $6 \mathrm{~min}$ and evaluated through time of immobility except for respiration.

\section{Memory-related tests}

Novel-object recognition: Novel-object recognition assay was carried out as described previously ${ }^{16-18}$. Two identical cubes were placed at the right and left corners of the apparatus. Mice were placed at the mid-point of the wall opposite the objects. After exposure for $10 \mathrm{~min}$, mice were remove from the apparatus and have a rest for $1 \mathrm{~h}$. One familiar object was placed at one corner of the apparatus, and one novel object was placed at the other corner. The animals were placed back into the apparatus and the duration of direct contact with two objects was recorded.

Social transmission of food preference (STFP): STFP assay was performed as described previously ${ }^{17,18}$. In this test, each mouse (observer) was fasted for $16 \mathrm{~h}$ in a single cage before test. Another female mouse, unfamiliar with the "observer", was fed with cocaine for $30 \mathrm{~min}$ as "demonstrator". After $30 \mathrm{~min}$ interaction time between "observer" and "demonstrator", "observers" were fed with two types of food containing cocaine or cinnamon. The consumption of cocaine- or cinnamon-containing food was measured to test the memory ability. Normal mice preferred eating food containing cocaine instead of cinnamon because of the memory of the scent.

\section{Reduced representation bisulfite sequencing}

Reduced representation bisulfite sequencing (RRBS) libraries were generated according to the previous study ${ }^{19}$. Genomic DNA (200 ng) was digested by Msp I to generate short fragments in a methylation-insensitive manner. After the digested DNA fragments were end-repaired and A-tailed, they were ligated to methylated Illumina adapters using Truseq DNA Sample Prep Kit (Illumina) following the user manual. The ligation products at the range of $150-700 \mathrm{bp}$ were selected to perform bisulfite conversion using MethylCode ${ }^{\mathrm{TM}}$ Bisulfite Conversion Kit (Life Technologies). Conversion products were PCRamplified using uracil + PCR polymerase (Zymo). The libraries were sequenced on an Illumina Hiseq $\mathrm{X}$ Ten platform under control of Hiseq Control Software.

\section{Primary neuron isolation and culture}

Hippocampus were dissected from E16 mouse embryos in DMEM-HG (HyClone) at $4{ }^{\circ} \mathrm{C}$. After removal of all the meninges, the tissues were digested with the digest 
medium ( $1 \mathrm{mg} / \mathrm{ml}$ papain, $5 \mathrm{mM}$ EDTA, DNase I $0.1 \mathrm{mg} /$ $\mathrm{ml}$ ) at $37^{\circ} \mathrm{C}$ for $30 \mathrm{~min}$. The supernatant was removed and the tissues were re-suspended in the digest medium. After stop the reaction with FBS, cells were plated onto poly-Dlysine-coated plates. After culturing with plating medium (Neurobasal ${ }^{\circledast}$ Medium, 5\% FBS, Supplement B27, GlutaMax I, Gentamycin) for $6 \mathrm{~h}$, the cells were cultured in the culture medium (Neurobasal ${ }^{\circledR}$ Medium, Supplement B27, GlutaMax I, Gentamycin).

\section{Preparation of adeno-associated virus}

AAV-DNMT3A-N-dCas9 (AAV9, $1.38 \times 10^{13}$ genomic copies per ml), AAV-AAV-C-dCas9 (AAV9, $1.68 \times 10^{13}$ genomic copies per $\mathrm{ml}$ ) and AAV-5U6-sgRNAs-EGFP (AAV9, $1.31 \times 10^{13}$ genomic copies per $\mathrm{ml}$ ) were packaged and provided by Shanghai Taiting Biological Co., Ltd.

\section{Viral infection of mice}

Mice were infected with appropriate rAAVs cocktails according to guidelines of IACUC. Briefly, mice were anesthetized by intraperitoneal injection of $5 \%$ chloral hydrate $(200 \mu \mathrm{l} / 20 \mathrm{~g}$ body weight), and mounted in a stereotaxic holder in order to fix the skulls. To infect mouse brain, rAAVs mixtures (equal amount of AAVDNMT3A-N-dCas9, AAV- AAV-C-dCas9 and AAV5U6-sgRNAs-EGFP) were delivered by a Nanoject II
(Drummond) system. $500 \mathrm{nl}$ of mixtures were injected into the hippocampus (AP: $-1.8 \mathrm{~mm}, \mathrm{ML}: \pm 1.5 \mathrm{~mm}$, DV: $-1.5 \mathrm{~mm}$ and $-2 \mathrm{~mm}$ ).

\section{Quantification and statistical analysis}

Statistical analysis was performed using Graph Pad PRISM 7. Data are shown as the mean \pm s.e.m. unless otherwise stated. No statistical methods were used to predetermine sample size. Experimenters were blind during all behavioral tests.

\section{Results}

\section{Targeted DNA methylation at the Mecp2 promoter in} Neuro-2a cells

To study the role of $M E C P 2$ promoter methylation in the ASD pathogenesis, we utilized a dCas9-based DNA methylation-editing tool to specifically increase methylation at the transcriptional start site (TSS) of Mecp2. To enhance the targeting efficiency, we fused the catalytic domain of DNA methyltransferase DNMT3A (DNMT3ACD) and its co-factor DNMT3L in tandem to the $\mathrm{N}$-terminus of dCas $9^{20}$. A catalytic dead DNMT3ACD mutant was used as a control. We also constructed a multi-sgRNA vector that simultaneously expresses five sgRNAs targeting the $\sim 500 \mathrm{bp}$ TSS region of Mecp 2 (Fig. 1a). All sgRNAs were demonstrated to be active as

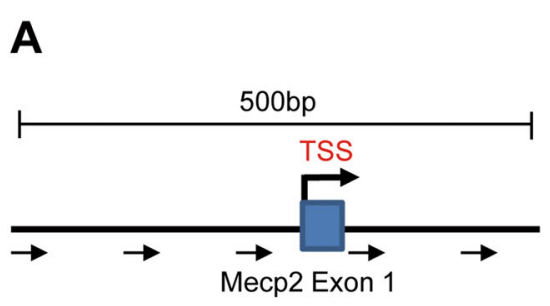

B

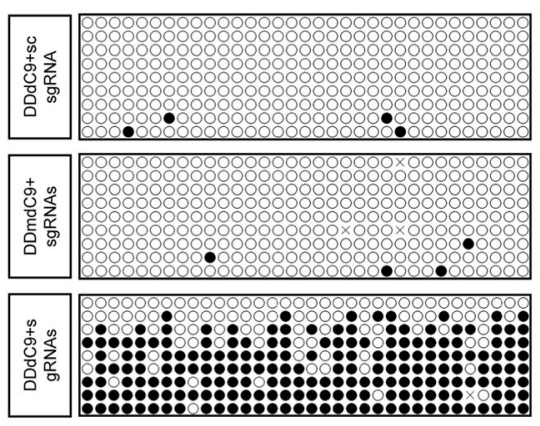

C

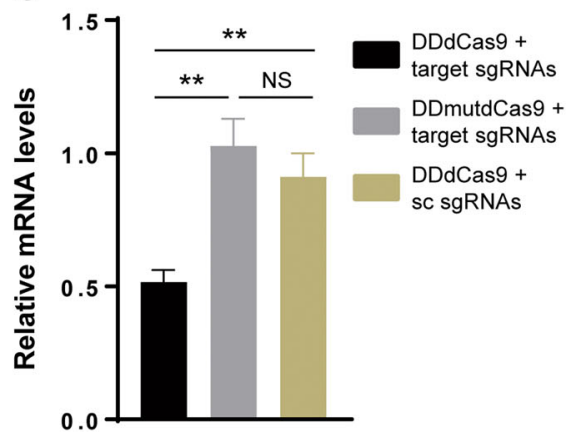

Fig. 1 Targeted DNA methylation of Mecp2 promoter in N-2a cells using DNMT3L-DNMT3A-dCas9. a Graphical representation of the Mecp2 locus showing sgRNAs target location. Transcription Start Site (TSS) is indicated by black arrow. The location of targets (1-5) for sgRNAs (Mecp2_1-5) are indicated by blue arrows. The Mecp2 exon is indicated by blue rectangle. $\mathbf{b}$ Representative methylation pattern as analyzed by bisulfite sequencing. c Targeted DNA methylation of Mecp2 promoter led to downregulation of Mecp2 expression in N2a cells. Expression of Mecp2 was calculated by quantitative PCR at 4 days after transfection. DDdCas9, DNMT3L-DNMT3A-dCas9; DDmutdCas9, DNMT3L-DNMT3Amut-dCas9; sC sgRNA, scrambled sgRNA. Data were statistically analyzed by Student's $t$ test $\left({ }^{* *} p<0.01\right.$; NS, no significance), and shown as the mean \pm s.e.m. ( $n=3$ from three independent experiments). 
they can induce site-specific DNA cleavage with CRISPRCas9 system (Fig. S1A, B).

To examine the targeted methylation of Mecp 2 promoter, we transfected Neuro-2a (N2a) cells with DNMT3LDNMT3A-dCas9 and multi-sgRNA vectors (referred hereafter as "methylation vectors"). At the same time, DNMT3L-DNMT3Amut-dCas9/multi-sgRNAs ("control vectors-1") and DNMT3L-DNMT3A-dCas9/scramblesgRNA ("control vectors-2") were used as two negative controls. The transfected cells were selected with $2 \mu \mathrm{g} / \mathrm{ml}$ puromycin and $20 \mu \mathrm{g} / \mathrm{ml}$ blasticidin for 2 days. The surviving cells were harvested to prepare for genomic DNA, and DNA methylation was analyzed by bisulfite sequencing. As expected, the overall methylation level at the Mecp 2 promoter was up-regulated to about $65 \%$ with the transfection of "methylation vectors", while the methylation level of this region was barely detectable with the treatment of "control vectors-1" or "control vectors-2" (Fig. 1b). Furthermore, Mecp 2 expression was significantly down-regulated as analyzed by qPCR (Fig. 1c).
To analyze the potential off-target effects, we collected $\mathrm{N} 2 \mathrm{a}$ cells at $48 \mathrm{~h}$ after transfection and performed reduced representation bisulfite sequencing (RRBS) analysis. We found that the global DNA methylation patterns, total methylation level, and methylation proportion were similar between the methylation and control groups (Fig. S2).

Furthermore, we measured the DNA methylation levels of 131 predicted off-target sites using bisulfite sequencing (Table S1). Only 4 out of 131 regions showed slightly higher levels of DNA methylation in the methylation group as compared to the controls (Table S1). Hence, our system represents a precise tool for locus-specific DNA methylation.

\section{Targeted Mecp2 promoter methylation down-regulates Mecp2 expression in mice}

For targeted methylation of Mecp 2 promoter in vivo, the "methylation vectors" or "control vectors-1" were injected into the mouse zygotes (Fig. 2a). For the

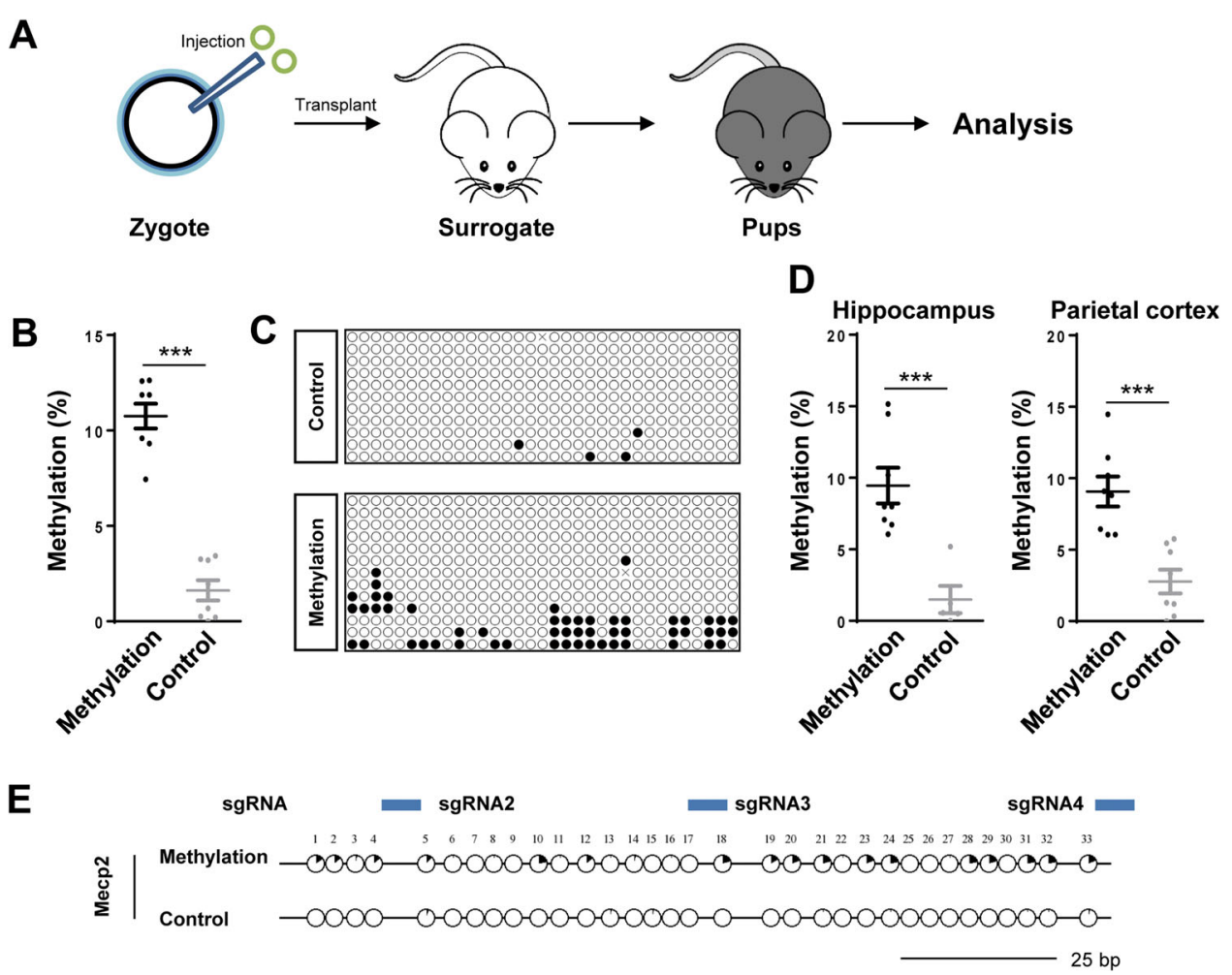

Fig. 2 Targeted DNA Methylation of Mecp2 in vivo Using DNMT3L-DNMT3A-dCas9. a Schematic illustration of experimental procedure for targeted DNA methylation in vivo. Constructs expressing DNMT3L-DNMT3A-dCas9 or DNMT3L-DNMT3Amut-dCas9 along with sgRNAs targeting the Mecp2 TSS region were pooled together and injected into mouse zygotes as indicated. $\mathbf{b}$ DNA methylation of targeted regions in the tail of male mice was analyzed by bisulfite sequencing. Each dot indicates one individual mouse. $n=8$ mice/group; Data were statistically analyzed by Student's $t$ test (*** $<0.001$ ), and shown as the mean \pm s.e.m. c Representative methylation pattern in the tails of male mice as determined by bisulfite sequencing. d DNA methylation of target regions in the hippocampus (left) and parietal cortex (right) of male mice were analyzed by bisulfite sequencing. Each dot indicates one individual mouse. $n=8$ mice/group; Data were statistically analyzed by Student's $t$ test $\left(^{* * *} p<0.001\right)$, and shown as the mean \pm s.e.m. e Methylation in the tail of male mice. Black portion of the circles indicates the methylation ratio in each CpG site. The results include all the sequencing data from mice described in (b). The Mecp2 sgRNAs (2-4) are indicated by blue rectangles. 


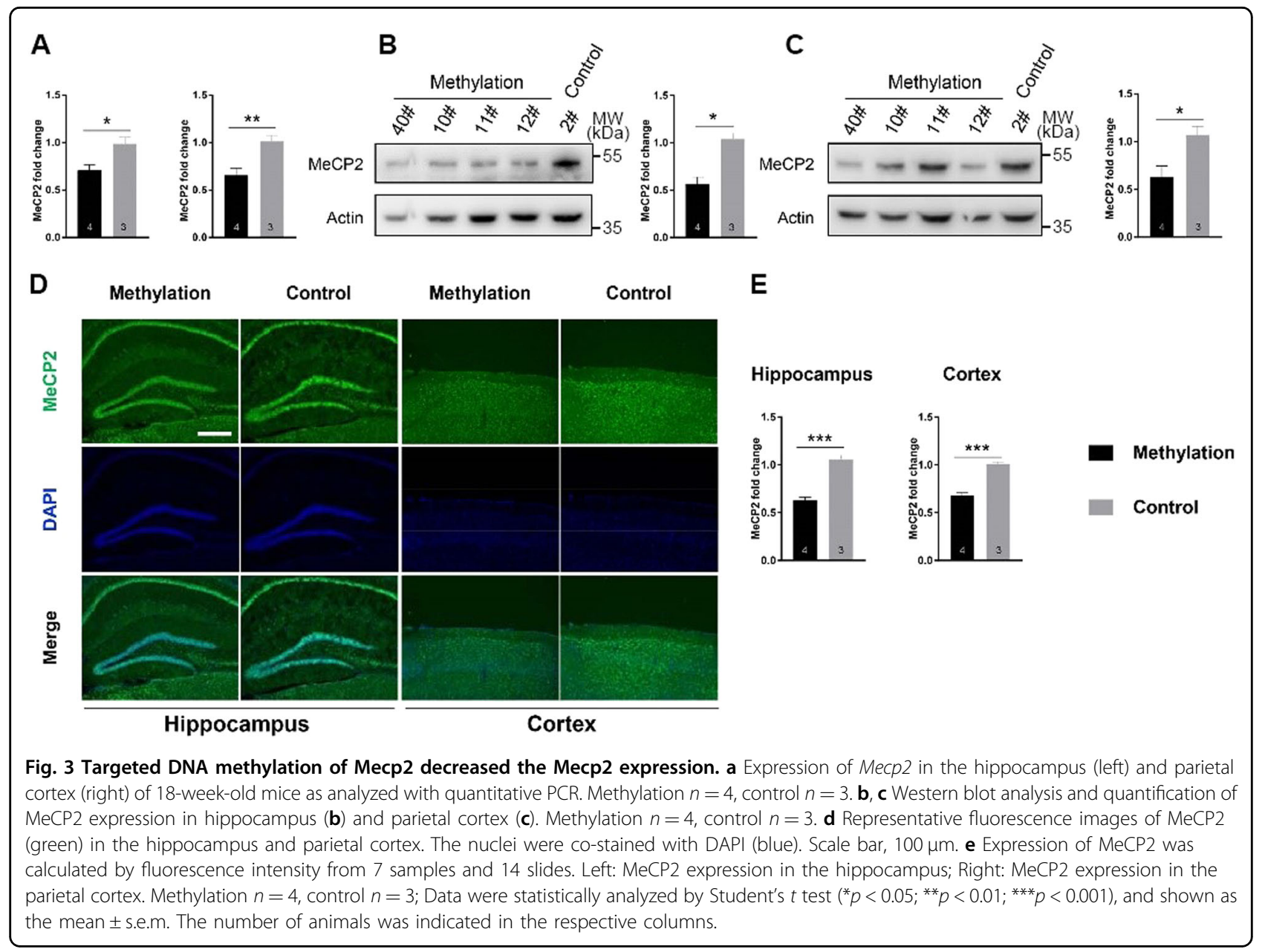

methylation group, we transferred 260 injected zygotes into 12 surrogate female mice, and obtained 61 live births (38 males and 23 females) (Table S2). For the control group, 248 injected zygotes were transferred into 10 surrogate female mice, and 65 live births (37 males and 28 females) were obtained (Table S2).

The genomic DNA from the tails of 3-week-old mice were subjected to bisulfate sequencing for analyzing the efficiency of the targeted DNA methylation. We found that male mice in the control group displayed less than 5\% methylation at the Mecp 2 promoter (Fig. 2b, c). By contrast, the males from the methylation group showed a significantly higher methylation at the target locus (Fig. 2b, c, e). As Mecp2 is an $\mathrm{X}$-linked gene, the basal DNA methylation in this region is quite high in female mice. There was no significant difference in the DNA methylation in females from control and methylation groups (Fig. S3).

We also determined the levels of targeted DNA methylation in a variety of tissues. As shown in Fig. 2d, the DNA methylation levels in the hippocampus and parietal cortex were significantly higher in the methylation group relative to the control group. (hippocampus: $9.5 \pm 3.0 \%$ vs.
$1.5 \pm 2.6 \%$; parietal cortex: $9.1 \pm 2.5 \%$ vs. $2.8 \% \pm 2.0 \%$; $p<$ 0.001 , unpaired $t$ test). In addition, the DNA methylation levels were also significantly elevated in the heart, liver, kidney, lung, and testis of mice from the methylation group (Fig. S4).

Consistent with the data from N2a cells, the transcription of Mecp 2 in the hippocampus and parietal cortex was significantly reduced in male mice from the methylation group, as compared with the control group (Fig. 3a). Furthermore, Western blot analysis showed significantly lower MeCP2 confirmed by the weak immunofluorescence staining of $\mathrm{MeCP} 2$ protein in tissue sections as compared to the control male mice (Fig. 3d, e).

\section{Targeted DNA methylation at Mecp2 promoter causes autism-like behaviors}

It is well known that abnormal $M E C P 2$ expression leads to ASD in human and ASD-like behaviors in mice ${ }^{11,21}$. In order to investigate whether manipulation of DNA methylation at the Mecp 2 promoter will cause abnormal behaviors in mice, we first examined the social interaction by using the three-chamber test. Prior to the social 


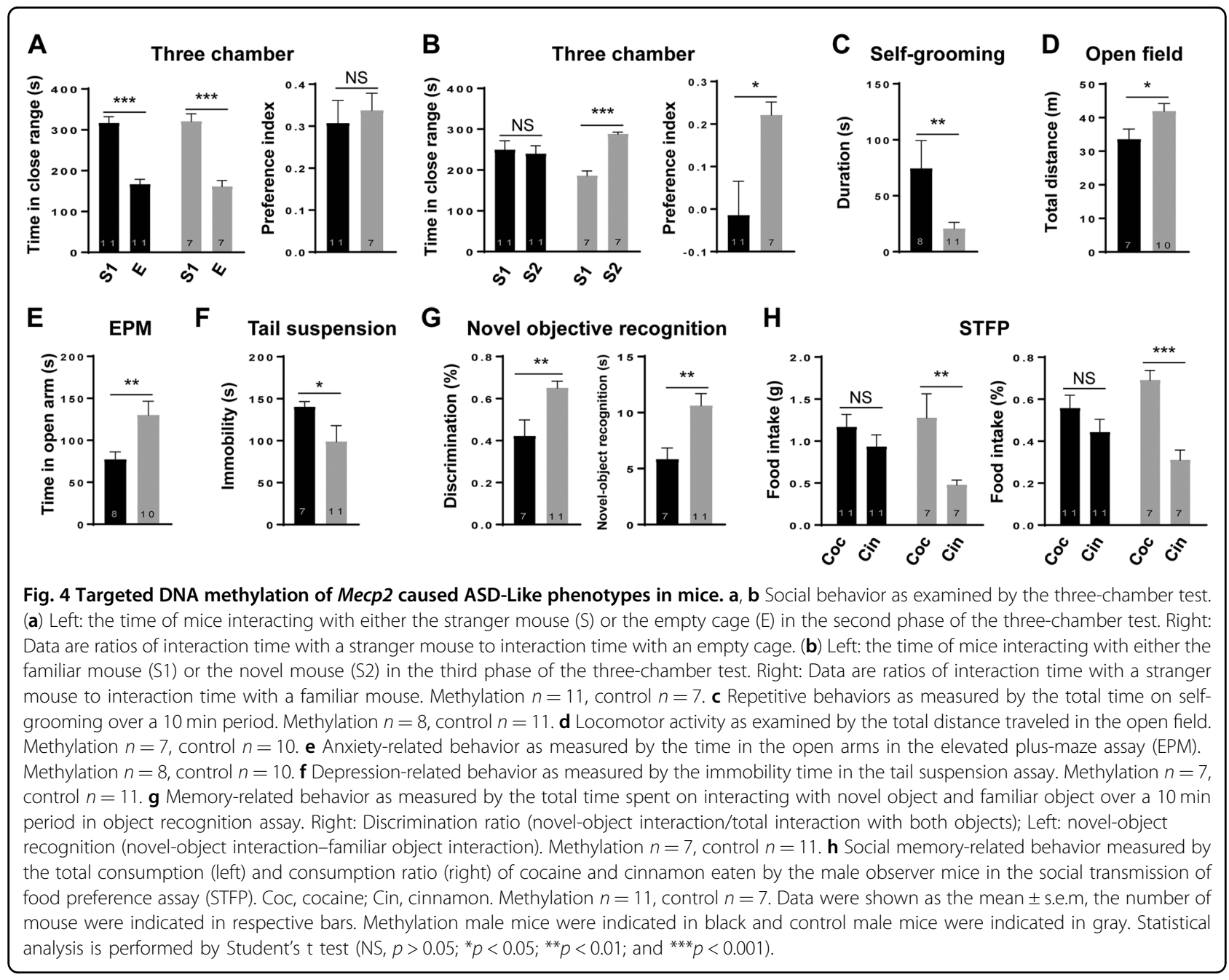

interaction, each mouse showed no preference in the chambers (data not shown). We found that mice from both methylation and control groups showed similar preference to the mouse over an object (Fig. 4a). When facing a familiar mouse versus an unfamiliar mouse, mice from the control group preferred to explore the unfamiliar mice, whereas mice from the methylation group showed no preference between familiar and unfamiliar mice (Fig. 4b). This result indicates that mice from the methylation group displayed a reduction in conspecific recognition or in their interest for social novelty compared with the control mice. Furthermore, we found that mice from the methylation group spent significantly longer time in self-grooming (Fig. 4c), reflecting an increased repetitive behavior.

Rett syndrome patients with MECP2 mutation also display reduced locomotion and anxiety ${ }^{11,22}$. We thus examined the locomotor activity of the mice by the open field test. We found that mice from the methylation group traveled a shorter total distance in the open field as compared to the control mice (Fig. 4d). Furthermore, mice from the methylation group had higher anxiety as they spent significantly less time in the open arms as compared to the control mice in the elevated plus-maze (EPM) test (Fig. 4e). In a tail suspension test, mice from the methylation group exhibited higher immobility time as compared to the control mice (Fig. 4f), indicating an elevated depression-like behavior in these mice.

Finally, we performed a series of behavioral tests to examine the memory- and cognition-related behaviors. In the novel-object recognition test, mice in the methylation group spent less time playing with a novel object relative to a familiar one than the control mice (Fig. 4g), suggesting deficiency in memory retention or reduced novelty seeking. Furthermore, the social transmission of food preference (STFP) test showed that mice of the methylation group failed to acquire the food preference of another mouse following 30-min co-housing in the 
absence of the food (Fig. 4h). Taken together, our data indicated that promoter-specific DNA methylation of Mecp2 gene could lead to behavioral alterations similar to those caused by Mecp 2 deficiency.

\section{Mecp2 promoter methylation in the hippocampus leads to defective social behaviors}

To determine whether Mecp 2 promoter methylation in the brain is sufficient to alter the behaviors of mouse, we set out to perform epigenetic modification in specific brain regions. To this end, we developed the AAV-based methylation vectors that target the TSS of Mecp 2 gene (Fig. 5a). Neurons infected with these viruses showed a significantly higher level of methylation in Mecp2 TSS region $(\sim 14 \%)$ as compared to neurons infected with the control vector ( 1\%) (Fig. 5b).

Given the importance of the hippocampus in memory and cognitive behaviors, AAV-based methylation vectors were bilaterally injected into the hippocampus of adult mice (Fig. 5c). The infection efficiency was verified in brain sections at two weeks after injection, and all behavioral tests were then performed at 4 weeks after injection. As shown in Fig. 5d-h, we found similar abnormality in social interaction in three-chamber test, increased anxiety on elevated open arm, novel-object recognition, and acquisition of food preference in STFP test, although the degrees of abnormality were lower than those found for mice with zygotebased methylation targeting. By contrast, we found no significant abnormality in locomotive and grooming activities, and depression-like behavior (Fig. S5). These results support the notion that specific methylation of Mecp 2 promoter in the brain could lead to ASD-like behaviors.

\section{Discussion}

In this study, we demonstrated a critical role of Mecp 2 promotor methylation in the ASD pathogenesis, by locusspecific methylation of Mecp2 promotor using an improved dCas9-based methylation targeting method. Methylation of Mecp 2 promotor led to reduction in Mecp2 gene expression, and alteration of a panel of behaviors mimicking the ASD symptoms. We further showed that specifically increasing the Mecp 2 promoter methylation in the hippocampus was sufficient to induce most of the behavioral changes.

DNA methylation on cytosine of a gene promoter usually leads to the silencing of gene expression. Abnormal DNA methylation has been observed in cancer and neurological disorders ${ }^{23}$. The functional significance of DNA methylation has been exploited by using transgenic mice with disruption of specific genes involved in the DNA (de)methylation, or DNMTs inhibitors, such as azacitidine and decitabine. However, such approach can only be applied non-selectively at the global genome level, but not at specific $\operatorname{loci}^{23}$.
The recently developed genome-modifying tools, such as zinc fingers (ZFs), transcription activator-like effectors (TALEs), or the CRISPR/Cas9 system have been successfully applied to loci-specific epigenome editing ${ }^{23,24}$. Several groups successfully repurposing the CRISPR-Cas9 system for targeted DNA methylation ${ }^{25-30}$. In this study, we further improved the targeting efficiency of the dCas9based vector by adding a sequence encoding DNMT3L, which functions as a co-factor of DNMT3A to enhance DNA methylation. As a result, the locus-specific methylation of Mecp2 promotor down-regulated Mecp2 expression, and mice with $M e c p 2$ promoter methylation exhibited ASD-like phenotypes. Our study thus provides the first evidence to support the causative relationship between DNA methylation and ASD-like phenotypes.

Most of the behaviors from mice with Mecp2 promoter methylation were in agreement with previous findings from Mecp2-deficient mice. However, there are contradict data in the anxiety-related behaviors by using different strains of Mecp2-deficient mice ${ }^{24,31-33}$. In our study, increasing Mecp2 promoter methylation led to elevated anxiety levels. Furthermore, hippocampus-specific Mecp2 promoter methylation also induced increased anxiety, reduced social interaction, and defective memory, which is consistent with the results from hippocampus-specific Mecp2 knockout mice $^{34}$. Nevertheless, no significant abnormality in locomotive and grooming activities, and depression-like behavior were observed in the mice with hippocampus-specific Mecp 2 promoter methylation, implying these behaviors may be related to other brain areas other than hippocampus.

Loci-specific epigenome editing not only help understand the causative relationship between DNA methylation in a specific DNA loci and pathological situations, but also have potential therapeutic applications. Indeed, Choudhury et al. used dCas9-TET1 fusion to selectively de-methylate targeted regions within BRCA1 promoter as directed by the designed single-guide RNAs (sgRNA), leading to the transcriptional up-regulation of the gene ${ }^{35}$. Recently, Liu et al. demonstrated that a locus-specific demethylation of Fragile $\mathrm{X}$ mental retardation (FMR1) gene, a putative fragile $\mathrm{X}$ syndrome (FXS)-causative gene, could correct the phenotype of FXS patient-derived induced pluripotent stem cells $^{30}$. Xu et al. developed a high-fidelity CRISPR/Cas9based gene-specific dioxygenase to rescue gene expression in vitro and attenuate renal fibrosis in vivo ${ }^{36}$. In this study, by targeted editing of DNA methylation, we demonstrated that methylation at the Mecp 2 promoter is sufficient to down-regulated Mecp2 expression and induce autism-like behaviors in mice. Further, Mecp 2 methylation specifically in the hippocampus is able to induce most of the behavioral alteration. Our finding therefore demonstrated DNA methylation of Mecp 2 promoter plays a causative role in autism-related symptoms, warranted potential drug discovery targeting such epigenetic alteration. 


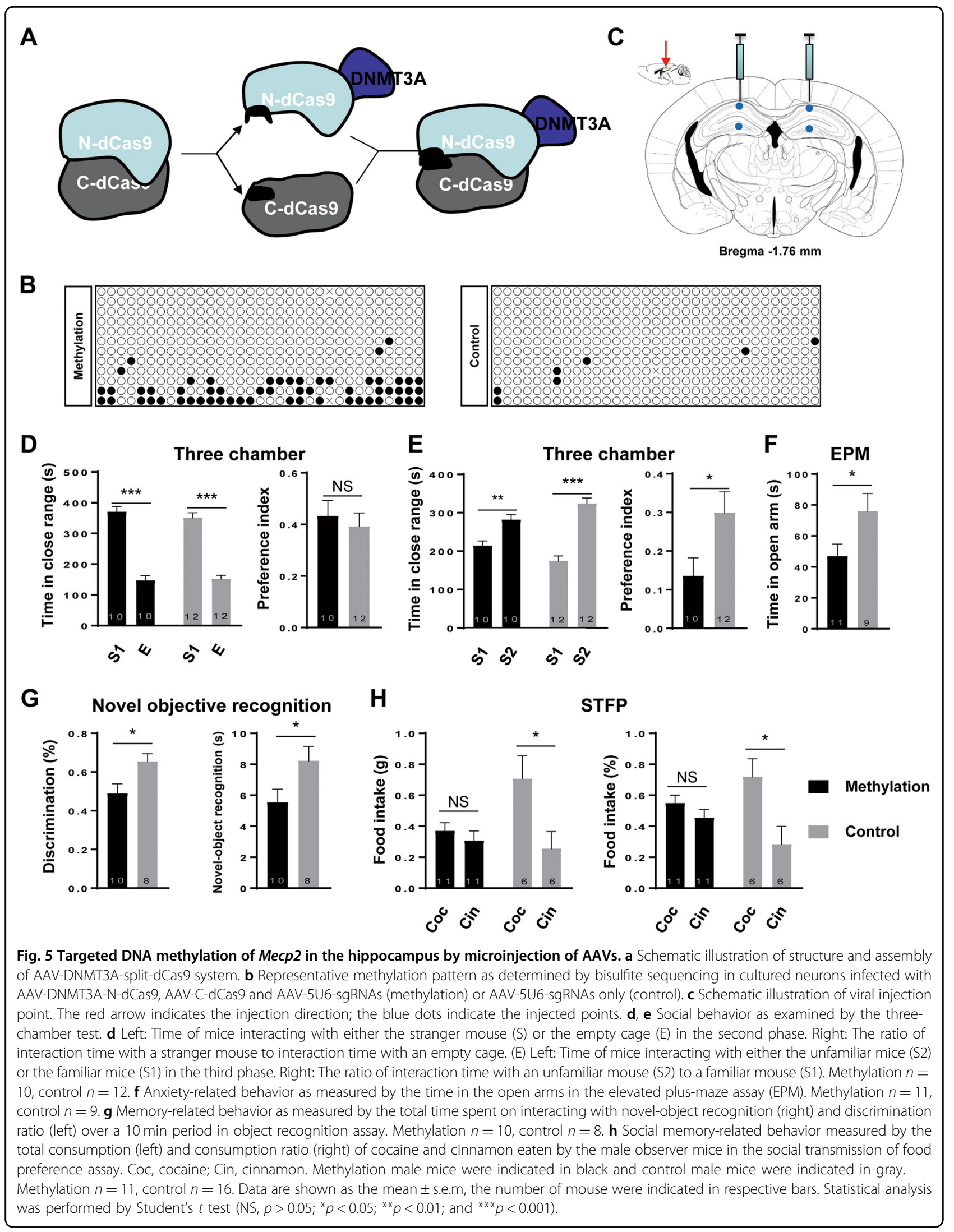




\section{Acknowledgements}

We thank members of Huang, Sun and Li labs for helpful discussions. We also thank Dr. Peng Cao for experimental technical support of STFP. We are grateful to Dr. Muming Poo from Institute of Neuroscience, CAS Center for Excellence in Brain Science and Intelligence Technology, Shanghai Institutes for Biological Sciences for critical revision and excellent manuscript editing. This work is supported by National Postdoctoral Program for Innovative Talents (BX201700266) to Z.L., CAS Key Technology Talent Program to Q.S., Shanghai Municipal Government Bureau of Science and Technology Grant (16JC1420200) to M.P. and Q.S; and National Science Foundation of China grants $(81770780,81728013,81671516)$, the Key research and development programs from Hunan Province (2018DK2010, 2018DK2013) to J.-D.L. as well as a Local Grant (17JC1420103).

\section{Author details}

'School of Life Science and Technology, ShanghaiTech University, 100 Haike Rd., Pudong New Area, Shanghai 201210, China. ${ }^{2}$ CAS Center for Excellence in Molecular Cell Science, Shanghai Institute of Biochemistry and Cell Biology, Chinese Academy of Sciences, University of Chinese Academy of Sciences, 320 Yueyang Road, Shanghai 200031, China. ${ }^{3}$ University of Chinese Academy of Sciences, 100049 Beijing, China. Institute of Neuroscience, Chinese Academy of Sciences (CAS) Key Laboratory of Primate Neurobiology, CAS Center for Excellence in Brain Science and Intelligence Technology, Shanghai Institutes for Biological Sciences, CAS, Shanghai 200031, China. ${ }^{5}$ Hunan Key Laboratory of Animal Models for Human Genetics, School of Life Sciences, Central South University, 110 Xiangya Road, Changsha 410078, China

\section{Data availability}

High-throughput sequencing data have been deposited in the NCBI Sequence Read Archive database with an accession code (SRP234617). Plasmids were deposited in Addgene with following catalog numbers: pGL3-U6-sgRNA (Addgene, 51133), pSt1374-N-NLS-DNMT3L-L-DNMT3A-Ldcas9-NLS (Addgene, 112209), pSt1374-N-NLS-DNMT3L-L-DNMT3Amut-L-dcas9-NLS (Addgene, 112210), pAAV-CAG-C-intein-C-spC9-H840AN863A-2xNLS-hGH (Addgene, 112211), pAAV-CAG-NLS-DNMT3A-L-N-spC9-Nintein-hG (Addgene, 112212), pAAV-5U6- sgRNAs-hsyn-EGFP (Addgene, 112213), pSt1374m-EF1alpha-N-NLSDNMT3L-LDNMT3A-L-dcas9 -NLS (Addgene, 112214) and pSt1374m-EF1alphaN-NLS-DNMT3L-L-DNMT3Amut-L-dcas9-NLS (Addgene, 112215).

\section{Conflict of interest}

The authors declare that they have no conflict of interest.

\section{Publisher's note}

Springer Nature remains neutral with regard to jurisdictional claims in published maps and institutional affiliations.

Supplementary Information accompanies this paper at (https://doi.org/ 10.1038/s41419-020-2290-x).

Received: 1 October 2019 Revised: 15 January 2020 Accepted: 21 January 2020

Published online: 03 February 2020

\section{References}

1. Woodbury-Smith, M. \& Scherer, S. W. Progress in the genetics of autism spectrum disorder. Dev. Med. Child Neurol. 60, 445-451 (2018).

2. Basu, S. N., Kollu, R. \& Banerjee-Basu, S. AutDB: a gene reference resource for autism research. Nucleic Acids Res. 37, D832-D836 (2009).

3. Pacchierotti, F. \& Spano, M. Environmental impact on DNA methylation in the germline: state of the art and gaps of knowledge. Biomed. Res. Int. 2015, 123484 (2015).

4. Wong, C. C. et al. Methylomic analysis of monozygotic twins discordant for autism spectrum disorder and related behavioural traits. Mol. Psychiatry 19, 495-503 (2014)

5. Elagoz Yuksel, M., Yuceturk, B., Karatas, O. F., Ozen, M. \& Dogangun, B. The altered promoter methylation of oxytocin receptor gene in autism. J. Neurogenet. 30, 280-284 (2016).

6. Zhu, L. et al. Epigenetic dysregulation of SHANK3 in brain tissues from individuals with autism spectrum disorders. Hum. Mol. Genet. 23, 1563-1578 (2014).
7. Nagarajan, R. P., Hogart, A. R., Gwye, Y., Martin, M. R. \& LaSalle, J. M. Reduced MeCP2 expression is frequent in autism frontal cortex and correlates with aberrant MECP2 promoter methylation. Epigenetics 1, e1-e11 (2006).

8. Nagarajan, R. P. et al. MECP2 promoter methylation and X chromosome inactivation in autism. Autism Res. 1, 169-178 (2008).

9. Nichol Edamura, K. \& Pearson, C. E. DNA methylation and replication: implications for the "deletion hotspot" region of FMR1. Hum. Genet. 118, 301-304 (2005).

10. Stoger, R., Kajimura, T. M., Brown, W. T. \& Laird, C. D. Epigenetic variation illustrated by DNA methylation patterns of the fragile-X gene FMR1. Hum. Mol. Genet. 6, 1791-1801 (1997).

11. Moretti, P. \& Zoghbi, H. Y. MeCP2 dysfunction in Rett syndrome and related disorders. Curr. Opin. Genet. Dev. 16, 276-281 (2006).

12. Wen, $Z$. et al. Identification of autism-related MECP2 mutations by whole-exome sequencing and functional validation. Mol. Autism 8, 43 (2017).

13. Shen, B. et al. Generation of gene-modified mice via Cas9/RNA-mediated gene targeting. Cell Res. 23, 720-723 (2013).

14. Su, S. et al. CRISPR-Cas9 mediated efficient PD-1 disruption on human primary T cells from cancer patients. Sci. Rep. 6, 20070 (2016).

15. Neul, J. L. et al. Rett syndrome: revised diagnostic criteria and nomenclature Ann. Neurol. 68, 944-950 (2010).

16. Bevins, R. A. \& Besheer, J. Object recognition in rats and mice: a one-trial nonmatching-to-sample learning task to study 'recognition memory'. Nat. Protoc. 1. 1306-1311 (2006).

17. Liu, Z. et al. IGF1-dependent synaptic plasticity of mitral cells in olfactory memory during social learning. Neuron 95, 106-122 e105 (2017).

18. Galef, B. G.Jr. Social learning of food preferences in rodents: rapid appetitive learning. Curr. Protoc. Neurosci. https://doi.org/10.1002/0471142301. ns0805ds21 (2003).

19. $\mathrm{Gu}, \mathrm{H}$. et al. Preparation of reduced representation bisulfite sequencing libraries for genome-scale DNA methylation profiling. Nat. Protoc. 6, 468-481 (2011).

20. Siddique, A. N. et al. Targeted methylation and gene silencing of VEGF-A in human cells by using a designed Dnmt3a-Dnmt3L single-chain fusion protein with increased DNA methylation activity. J. Mol. Biol. 425, 479-491 (2013).

21. Qiu, Z. Deciphering MECP2-associated disorders: disrupted circuits and the hope for repair. Curr. Opin. Neurobiol. 48, 30-36 (2018).

22. Segawa, M. Discussant-pathophysiologies of Rett syndrome. Brain Dev. 23 (Suppl 1), S218-S223 (2001).

23. Shahbazian, M. et al. Mice with truncated MeCP2 recapitulate many Rett syndrome features and display hyperacetylation of histone H3. Neuron 35 243-254 (2002).

24. Samaco, R. C. et al. A partial loss of function allele of methyl-CpG-binding protein 2 predicts a human neurodevelopmental syndrome. Hum. Mol. Genet. 17, 1718-1727 (2008)

25. Lei, Y. et al. Targeted DNA methylation in vivo using an engineered dCas9MQ1 fusion protein. Nat. Commun. 8, 16026 (2017).

26. Morita, S. et al. Targeted DNA demethylation in vivo using dCas9-peptide repeat and scFv-TET1 catalytic domain fusions. Nat. Biotechnol. 34, 1060-1065 (2016).

27. Liu, X. S. et al. Editing DNA methylation in the mammalian genome. Cell 167, 233-247 e217 (2016).

28. Chedin, F., Lieber, M. R. \& Hsieh, C. L. The DNA methyltransferase-like protein DNMT3L stimulates de novo methylation by Dnmt3a. Proc. Natl Acad. Sci. USA 99, 16916-16921 (2002).

29. Gowher, H., Liebert, K., Hermann, A., Xu, G. \& Jeltsch, A. Mechanism of stimulation of catalytic activity of Dnmt3A and Dnmt3B DNA-(cytosineC5)-methyltransferases by Dnmt3L. J. Biol. Chem. 280, 13341-13348 (2005).

30. Liu, X. S. et al. Rescue of fragile $X$ syndrome neurons by DNA methylation editing of the FMR1 gene. Cell https://doi.org/10.1016/j.cell.2018.01.012 (2018).

31. Zhou, $H$. et al. Selective preservation of cholinergic MeCP2 rescues specific Rett-syndrome-like phenotypes in MeCP2(stop) mice. Behav. Brain Res. 322 , 51-59 (2017).

32. Gemelli, T. et al. Postnatal loss of methyl-CpG binding protein 2 in the forebrain is sufficient to mediate behavioral aspects of Rett syndrome in mice. Biol. Psychiatry 59, 468-476 (2006). 
33. Philippe, T. J. et al. Loss of MeCP2 in adult 5-HT neurons induces 5-HT1A autoreceptors, with opposite sex-dependent anxiety and depression phenotypes. Sci. Rep. 8, 5788 (2018).

34. Zhang, Y. et al. Loss of MeCP2 in cholinergic neurons causes part of RTTlike phenotypes via alpha7 receptor in hippocampus. Cell Res. 26, 728-742 (2016).
35. $\mathrm{Xu}, \mathrm{X}$. et al. High-fidelity CRISPR/Cas9- based gene-specific hydroxymethylation rescues gene expression and attenuates renal fibrosis. Nat. Commun. $\mathbf{9}$ 3509 (2018).

36. Li, Y. et al. CRISPR-dCas9 mediated TET1 targeting for selective DNA demethylation at BRCA1 promoter. CRISPR-dCas9 mediated TET1 targeting for selective DNA demethylation at BRCA1 promoter. Oncotarget. 7, 46545-46556 (2016). 